\title{
Generalized Approach to Translating Exercise Tests and Prescribing Exercise
}

\author{
Carl Foster ${ }^{1, *}$, James D. Anholm ${ }^{2}$, Daniel Bok ${ }^{3}{ }^{(}$, , Daniel Boullosa $^{4,5}{ }^{(0}$, Giancarlo Condello ${ }^{6}$, \\ Cristina Cortis ${ }^{7}{ }^{10}$, Andrea Fusco ${ }^{7}\left(0\right.$, Salvador J. Jaime ${ }^{1}$, Jos J. de Koning ${ }^{1,8}$, Alejandro Lucia ${ }^{9,10}$, \\ John P. Porcari ${ }^{1}$, Kim Radtke ${ }^{1}$ and Jose A. Rodriguez-Marroyo ${ }^{11}$
}

1 Department of Exercise and Sport Science, University of Wisconsin-La Crosse, La Crosse, WI 54601, USA; sjaime@uwlax.edu (S.J.J.); j.j.de.koning@vu.nl (J.J.d.K.); jporcari@uwlax.edu (J.P.P.); kradtke@uwlax.edu (K.R.) VA Medical Health Care System, Loma Linda, CA 92697, USA; James.Anholm@va.gov

Faculty of Kinesiology, University of Zagreb, 10000 Zagreb, Croatia; daniel.bok@kif.unizg.hr

4 INISA, Federal University of Mato Grosso do Sul, Campo Grande 79070-900, Brazil; daniel.boullosa@gmail.com

5 College of Healthcare Sciences, James Cook University, Townsville 4811, Australia

6 Graduate Institute of Sports Training, Institute of Sports Sciences, University of Taipei, Taipei 111, Taiwan; giancarlo.condello@gmail.com

7 Department of Human Sciences, Society and Health, University of Cassino and Lazio Meridionale, 03043 Cassino, Italy; c.cortis@unicas.it (C.C.); andrea.fusco@unicas.it (A.F.)

8 Department of Human Movement Science, Movement Sciences Amsterdam, Vrije Universiteit, 1081BT Amsterdam, The Netherlands

9 Faculty of Sport Sciences, Universidad Europea de Madrid, 28670 Villaviciosa de Odón, Spain; alejandro.lucia@universidadeuropea.es

10 Research Institute 'imas12', Hospital 12 de Octubre, 28041 Madrid, Spain

11 Department of Physical Education and Sports, University of León, 24071 León, Spain; j.marroyo@unileon.es

* Correspondence: cfoster@uwlax.edu

Received: 11 July 2020; Accepted: 10 August 2020; Published: 12 August 2020

\begin{abstract}
Although there is evidence supporting the benefit of regular exercise, and recommendations about exercise and physical activity, the process of individually prescribing exercise following exercise testing is more difficult. Guidelines like \% heart rate (HR) reserve (HRR) require an anchoring maximal test and do not always provide a homogenous training experience. When prescribing HR on the basis of \% HRR, rating of perceived exertion or Talk Test, cardiovascular/perceptual drift during sustained exercise makes prescription of the actual workload difficult. To overcome this issue, we have demonstrated a strategy for "translating" exercise test responses to steady state exercise training on the basis of \% HRR or the Talk Test that appeared adequate for individuals ranging from cardiac patients to athletes. However, these methods depended on the nature of the exercise test details. In this viewpoint, we combine these data with workload expressed as Metabolic Equivalent Task (METs). We demonstrate that there is a regular stepdown between the METs during training to achieve the same degree of homeostatic disturbance during testing. The relationship was linear, was highly-correlated $(r=0.89)$, and averaged $71.8 \%$ (Training METs/Test METs). We conclude that it appears possible to generate a generalized approach to correctly translate exercise test responses to exercise training.
\end{abstract}

Keywords: exercise prescription; target heart rate; RPE; Talk Test 


\section{Introduction}

Exercise is a very positive health behavior. As far back as Hippocrates in the 4th century Before Common Era (BCE) and Galen in the 3rd century BCE, the concept of mens sana in corpore sano "a healthy mind in a healthy body" has been one of the cornerstones of medical practice. Exercise is particularly beneficial considering that in most of the developed world, heart disease is the leading attributable cause of death ( $45-50 \%$ of deaths), and cancer the 2 nd (30-35\% of deaths), although their incidence is meaningfully reduced in people who follow out genetic heritage and perform a large volume of exercise [1]. Despite the development of significant diagnostic and therapeutic medical options, the incidence of both diseases rose steadily through the first 70 years of the 20th century and began to decline after the 1962 publication of the United States Surgeon General's recommendations against smoking [2] and Cooper's Aerobics in 1968 [3], which essentially launched the "jogging" movement. The evidence supporting the positive health benefit of regular exercise is based on an abundant data base of epidemiological studies [4-8]. More recent population studies have demonstrated a dose-response effect of exercise on the risk of mortality, with an apparent saturation of benefit at $\sim 20$ Metabolic Equivalent Task (MET) hours per week [9-14]. There is, paradoxically, a slight excess in mortality above $\sim 75$ MET hours per week [9-11]. This is paralleled by a progressive reduction in the probability of cardiovascular events with increases in the number of steps accumulated per day [12-14].

Table 1. Negative outcomes during exercise training.

\begin{tabular}{l}
\hline Congenital Abnormalities \\
\hline Hypertrophic cardiomyopathy \\
Arrhythmogenic RV dysplasia \\
Coronary artery anomalies \\
\hline Undiagnosed Coronary Artery Disease \\
\hline Pre-exercise screening identifies $>50 \%$ \\
First presentation of cardiovascular disease is often fatal \\
$33 \%$ males, $12 \%$ females \\
\hline Drug Use \\
\hline Anabolic steroids \\
Stimulants \\
Erythropoietin \\
Recreational drugs \\
\hline Trauma \\
\hline Commotio cordis \\
\hline
\end{tabular}

With any health/medical therapy, particularly with drugs and surgery, there are usually iatrogenic (negative) side effects that need to be accounted for, which influences the dose of exercise recommended. In the 19th century, exercise (particularly athletics) was often viewed with some suspicion [15-17]. A series of reports, based on data collected after the beginning of the "jogging revolution" following the 1968 publication of Cooper's Aerobics, suggested that middle-aged joggers were at somewhat of an increased risk during exercise [17]. This risk was largely seen to be based on a changed flow-demand relationship in the coronary circulation and the risk of rupturing of existing atherosclerotic plaque. Negative outcomes during exercise training appear to be based on a variety of factors (Table 1) [18]. In adults, early studies suggested that bad outcomes during exercise were most often seen in persons with underling coronary artery disease [17], and that the presentation of myocardial infarction vs. sudden cardiac death seemed to be related to the presence/absence of prior myocardial infarction (e.g., prior myocardial scarring) [17]. In the 1990s, a series of studies focused on the "triggering" of myocardial infarction, noted that when exercise was involved, it was usually "unaccustomed heavy ( $>6$ METs) exercise" in previously sedentary individuals" [17]. Across time, the risk of untoward 
events during exercise training, whether in healthy individuals or patients with known cardiovascular disease, has generally decreased [15], perhaps largely because we have become better at recognizing when not to begin an exercise program, and by recognizing the importance of controlling intensity during the early days/weeks of an exercise program [15].

\section{Exercise Testing}

Graded exercise testing is a fundamental technique within medical diagnostics, fitness assessment, performance diagnostics, and exercise prescription [18-20]. There are a variety of reasons for exercise testing (Table 2) [18]. Exercise testing, which at the minimum usually involves progressively harder exercise, with electrocardiogram (ECG) and hemodynamic monitoring, can be augmented in a variety of ways including measurement of respiratory gas exchange, and methods designed to measure myocardial perfusion or ventricular function [19]. Exercise capacity derived from graded exercise tests has been shown to be very useful in terms of defining prognosis [21,22]. In middle-aged individuals, including patients with known cardiovascular disease, peak exercise capacities of $>8$ METs are associated with 5-year survival of $\sim 95 \%$, which gives physicians the latitude to try less invasive therapies. From the standpoint of prescribing exercise, there is a long tradition of using either the relative heart rate or heart rate reserve, or a normalized approach to exercise capacity (e.g., \% heart rate (HR) reserve (\% HRR), or \% maximal oxygen consumption (\% $\mathrm{VO}_{2}$ max) or maximal METs) $[18,19]$. For example, in two patients with the same evidence of a disease process (e.g., ST segment depression on the ECG, correlated with chest pain) may be viewed, and treated, very differently depending on their prognosis estimated from exercise capacity.

Table 2. Reasons for exercise testing.

\begin{tabular}{l}
\hline Evaluate Exertional Discomfort \\
\hline Reduced exercise tolerance \\
Chest pain \\
Dyspnea \\
Claudication \\
Cerebral symptoms \\
\hline Reveal Occult Pathology \\
\hline Change presentation of cardiovascular disease \\
\hline Define Prognosis \\
\hline Guide to exercise prescription strategy \\
\hline Exercise Prescription \\
\hline Relative percentage concept \\
Ischemic/arrhythmic threshold \\
\hline
\end{tabular}

In most cases, maximal exercise is often employed to allow optimization of the diagnostic sensitivity of exercise testing [23] and anchoring of the exercise prescription. However, submaximal testing has been shown to be a valuable alternative when maximal testing is not possible [24] and is certainly less demanding for the patient, may be perceived as safer, and does not require physician involvement. Submaximal exercise outcomes, such as the ventilatory threshold (VT), have become recognized as effective criteria for sustainable exercise capacity $[25,26]$. Exercise testing in clinical populations is generally thought to be quite safe, with complications requiring medical intervention occurring in $\sim 1 \%$ of tests [27] and in healthy individuals/athletes is nearly zero [28]. More recent approaches to using the Rating of Perceived Exertion (RPE) [24,29] and the Talk Test [24,30] to evaluate exercise capacity have appeared and may be just as effective for exercise prescription. For example, an RPE rating of 13-14 or the first time speech comfort is "equivocal" is very close to the VT [24,30]. 
To the degree that VT may be just as good of an index of sustainable exercise capacity as $\mathrm{VO}_{2}$ max, such submaximal exercise testing options hold great promise.

\section{Exercise Advice vs. Prescription}

The value of exercise as a health promoting behavior is large enough that professional societies such as the American College of Sports Medicine, the American Heart Association, and the Centers for Disease Control and Prevention have issued guidelines for public behavior [31,32]. These guidelines, which carry a very favorable benefit-risk ratio, recommend that all adults accumulate at least $150 \mathrm{~min}$ of moderate (almost always $<$ VT) intensity exercise, preferably distributed over at least 5 days per week. If one includes ordinary activities as well, this would represent $~ 10,000$ steps per day [12-14] or $~ 20$ METs hours per week [9-11]. It is important to note that these levels of recommendation, which probably approximate $7 \mathrm{~h}$ per week, are in excess of the $150 \mathrm{~min}$ per week recommended by professional societies [31,32]. The difference may be based on the belief that compliance to recommendations of $150 \mathrm{~min}$ per week is likely to be higher than to $>400 \mathrm{~min}$ per week, and that the public health benefit of more people doing less than idealized exercise is larger than better grounded recommendations which may have lower compliance. These recommendations are not very individually tailored. In many ways, they are comparable to the traditional health recommendations, "an apple a day keeps the doctor away", but have a likely large benefit in terms of health risk, with very minimal likelihood of untoward complications.

However, many people prefer more individually driven exercise prescription. Or in individuals with more fragile clinical conditions, more individually specific advice might be of value. Consistent with the concept of Exercise is Medicine ${ }^{\circledR}$ promoted by the American College of Sports Medicine, there would be some sort of exercise-based evaluation that would allow the generation of an individual exercise prescription. The concept of the American College of Sports Medicine is based on the FITT-VP concept (frequency, intensity, time, type, volume, and progression) [18]. The most difficult of these elements to prescribe is intensity. In the original concept, the intensity of exercise was prescribed based on $\%$ of maximal HR (\% HRmax), \% HRR, \% METs, or \% MET Reserve [18]. This practice was supported by an abundant data base from randomized trials. However, it requires the presence of a maximal exercise test to anchor the HRmax or max METs, particularly since age-based population estimates of HRmax are known to be individually inadequate [33]. As early as the late 1970s, there were also reports suggesting that exercise prescriptions built on the so-called "relative percent concept" were not very good at creating a homogenous training experience and response [34,35]. Beyond this, it is widely recognized that HRmax achieved during a single incremental bout of exercise (particularly during tests conducted for clinical diagnostics) is unlikely to represent a true HRmax achieved during field testing, or interval training. More recent recommendations have suggested that exercise prescription concepts based on ventilatory/lactate threshold might be superior $[25,26,36]$. However, determination of "threshold" is technologically demanding, and requires a maximal exercise test. Recent work from our laboratory has suggested that the Talk Test may offer a technologically simple approach to VT estimation [30]. In the Talk Test, it is common to have subjects recite a standard speech passage of $\sim 90$ words, and then respond to the question, "can you speak comfortably?". When the subject responds with "yes", they are typically below the intensity of the VT. The first equivocal response, "yes, but", usually occurs at about the intensity of the VT. If the subject responds with a definite "no", the intensity is typically close to the respiratory compensation threshold [30]. Further, work with the $\mathrm{RPE}$ has suggested a low-tech way to evaluating either $\mathrm{VT}$ or $\mathrm{VO}_{2}$ max and prescribing exercise that is useful for recommending the intensity of training $[25,37,38]$.

\section{Functional Translation}

One of the complicating issues when prescribing exercise training, particularly based on responses during submaximal or maximal exercise testing, is that the HR or RPE or Talk Test response at a particular workload during the exercise test "drifts" as exercise is sustained during training. This may 
be attributable to progressive changes in core temperature, to accumulation of catecholamines, to progressive dehydration, or to other factors related "to fatigue". In other words, a workload that elicits a HR of 130 during exercise testing may have a HR of 145 after $30 \mathrm{~min}$, secondary to cardiovascular drift. This leads to the practical problem of advising patients what to do in the gymnasium based on their exercise test responses. Imagine a person with a resting HR of 50, a maximal HR of 150, and thus a target HR at 70\% HRR of 120, with an RPE of 13 and a Talk Test response of "yes, I can talk comfortably". Let us say that this person achieves this HR at $6 \mathrm{~min}$ into a standard Bruce treadmill protocol $(2.5 \mathrm{mph}$ $(4.0 \mathrm{kmh}), 12 \%$ grade). However, if they go to the gymnasium and begin exercise training at this workload, their HR will quickly be greater than 120, their RPE will be 15 or more, they will not be comfortable talking, and they will have to quit after $15 \mathrm{~min}$ or so. Clearly, a prescribed workload will "drift" beyond the scope intended. This highlights the importance of monitoring responses during exercise training via multiple mechanisms (\% HRR, RPE, Talk Test). However, sometime the monitoring methods have enough lag that the beginning exerciser gets "behind the curve" and becomes overly fatigued. In an experienced exerciser, this is not a problem. However, in the more vulnerable exerciser, it could provide the substrate for (at least) an unpleasant exercise session, if not an untoward event. If the exercise test responses could be "translated" such that the target workload designed to achieve target values for HR, RPE and Talk Test could be adjusted (down regulated) before the session begins then the response during training might be more optimal.

We have taken this approach with ambulation following a standard treadmill test [38], with cycling after a standard cycle test [38], during arm-leg ergometry [39], during recreational activities [40] for target HR. We have also taken the same approach using the Talk Test as the outcome measure in sedentary individuals [41], well-trained individuals [42], and cardiac patients [30,43,44]. However, the magnitude of the "step down" (down regulation) in workload from exercise testing to exercise training is highly individual and depends on the details of the exercise test protocol. For example, in more rapidly ramped protocols, or in standard clinical protocols (e.g., Bruce treadmill protocols), the lack of near steady state conditions during testing makes the necessity for down-regulating the training session more difficult.

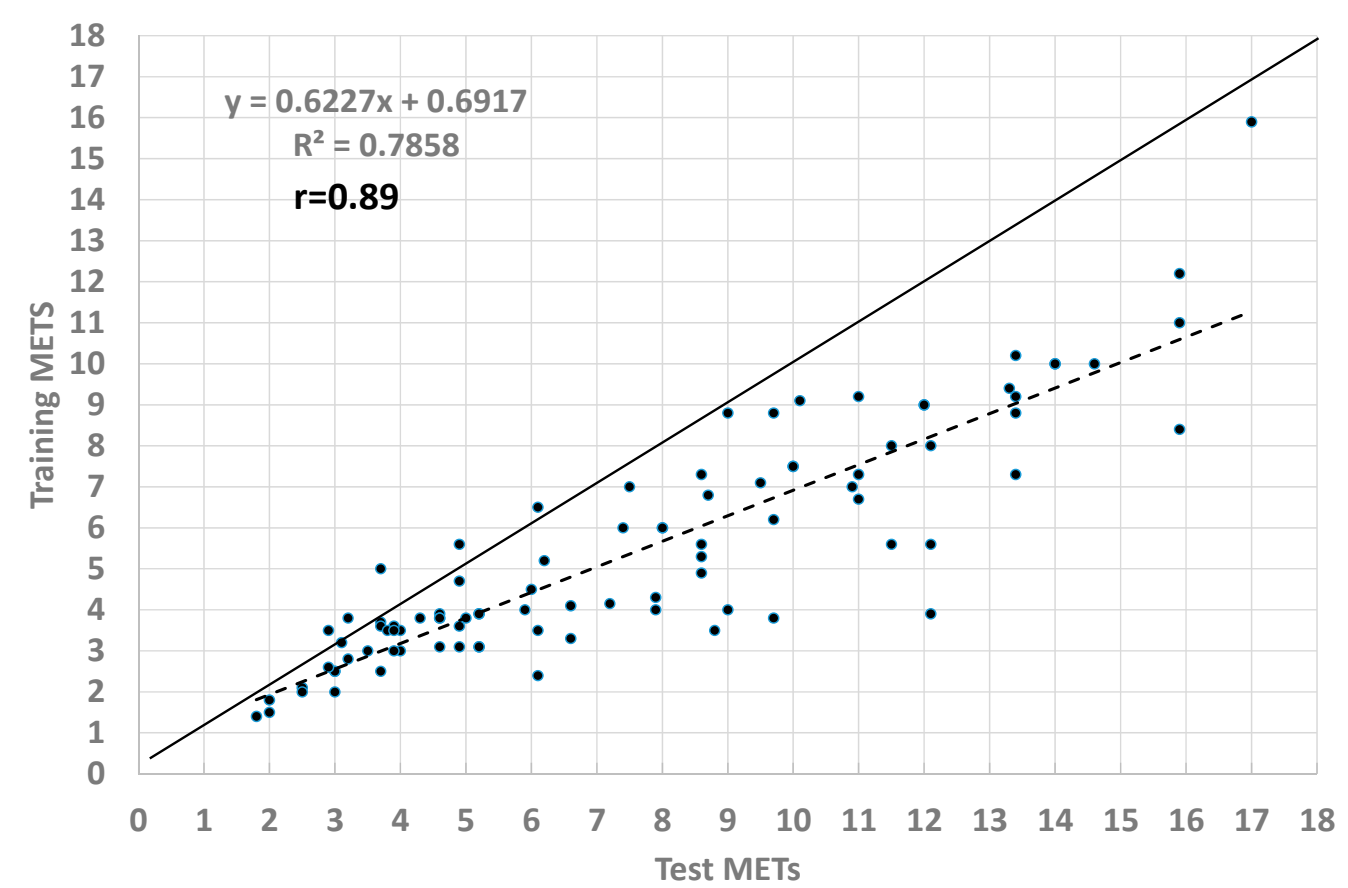

Figure 1. Comparison of exercise intensity (expressed as Metabolic Equivalent Task (METs) during exercise testing and exercise training with the same level of objective exercise intensity (\% of Heart Rate Reserve, Rating of Perceived Exertion, Talk Test) based on combined results from several studies [38-44]. 
In this paper, we have taken the strategy of trying to "generalize" the magnitude of step down, by computing the relative workload, expressed as METs, on the basis of standard metabolic formulas [18] during both exercise testing and training, based on our previously published data [38-44]. We then plotted the METs during the exercise test against the METs during the steady state period (15-30 min) during exercise training, with the same magnitude of homeostatic disturbance in terms of \% HRR, Talk Test score, and RPE. The results of this comparison are presented in Figure 1. The translated MET values were well correlated $(r=0.89)$ and the mean "step down" followed a linear regression, with an average step-down to $71.8 \%$ from testing $(7.66 \pm 4.03 \mathrm{METs})$ to training ( $5.50 \pm 2.92 \mathrm{METs})$. This strategy, of course, is primarily designed for near steady state training sessions. In principle it could be extrapolated to interval training, although factors such as the length of the hard and easy segments, as well as the difference between hard and easy segments would have to be considered. Accordingly, the purpose of this study was to develop a 'generalized model' for translating exercise test responses to exercise prescription.

\section{Discussion}

The main finding of this viewpoint was that it appears possible to generalize previous studies [38-44] intended to "translate" exercise test responses into exercise prescriptions, by expressing the workload as METs. Using this approach, it appears that steady state exercise training at $65-75 \%$ of the workload yielding a particular marker of exercise intensity (i.e., \% HRR, RPE, Talk Test) during exercise testing will yield comparable responses during exercise training. This problem has not otherwise been widely addressed. However, our results suggest that a larger "stepdown" than the $\sim 10 \%$ recommended by Mezzani et al. [24] is necessary. The results are also consistent with the finding of de Koning et al. [45] that VT occurs at about $50 \%$ of peak power output (usually $\sim 70 \% \mathrm{VO}_{2}$ max) and that most exercise training, whether for athletes [26] or non-athletes [19], takes place at intensities $<$ VT [20]. Validation of the magnitude of "stepdown" awaits further prospective data.

In the case of already active individuals, the spontaneous choice of the exercise level often yields intensities within commonly recommended guidelines [18]. Indeed, using the Talk Test as a surrogate of exercise intensity, athletic subjects required relatively little adjustment of workload beyond the first moments of the exercise bout $[30,42,43]$. In other words, in already experienced exercisers, finding the right intensity is relatively simple and rapid. Further, even in minimally trained individuals and cardiac patients, adjustment of the exercise level on the basis of speech comfort yielded appropriate relative MET and \% HRR values within a very few minutes of exercise [30,44]. We also know that previously untrained individuals can self-regulate exercise intensity using the RPE scale and achieve an effective training response [37].

In sedentary individuals, unaccustomed heavy exercise can be associated with the "triggering" of myocardial infarction [17]. However, we also know that the incidence of exercise related complications, both in healthy individuals and in cardiac rehabilitation programs, has decreased over time [15], most likely because we are better at regulating exercise intensity during the early weeks of new exercise programs (e.g., avoiding conditions that might promote "triggering" of myocardial infarction). Against the background that many people who have been sedentary for many years and who are beginning an exercise program later in life, and that they may tend to exercise at intensities recalled from earlier in their life. This workload may now be too strenuous and may predispose toward the development of untoward events during training. Thus, rather than prescribing exercise on the basis of \% HRR or even RPE, which may take some time to show that the exercise is too strenuous, it would seem to be desirable to have a mechanism for deciding what the appropriate workload should be during the first few days of training, and then fine tuning (e.g., triangulate) this intensity on the basis of conventional monitoring tools (\% HRR, RPE, Talk Test). 


\section{Conclusions}

The present results suggest the utility of "translating" exercise test responses into the workload during exercise training that will yield appropriate levels of exercise intensity as defined by \% HRR, $\mathrm{RPE}$, or Talk Test. It appears that reducing the workload to $65-75 \%$ of that during the exercise test is reasonable and can be done on the basis of METs calculated using standard methods [18]. Particularly in sedentary individuals beginning an exercise program or in patients during rehabilitation, this approach may yield useful estimates of exercise intensity and contribute to both the safety and efficacy of exercise therapy. These data suggest a simple approach to using exercise testing results, which are meaningfully generalized [38-44] from our earlier more specific results. Hopefully, this makes the "functional translation" approach easier to use, although this new approach requires future experimental verification.

Author Contributions: Conceptualization, C.F., J.D.A., D.B.(Daniel Bok), D.B. (Daniel Boullosa), C.C., A.F., J.J.d.K., J.P.P., and J.A.R.-M.; writing-original draft preparation, C.F., G.C., S.J.J., A.L., and K.R.; writing-review and editing, C.F., J.D.A., D.B.(Daniel Bok), D.B. (Daniel Boullosa), G.C., C.C., A.F., S.J.J., J.J.D.K., A.L., J.P.P., K.R., and J.A.R.-M. All authors have read and agreed to the published version of the manuscript.

Funding: This research received no external funding.

Conflicts of Interest: The authors declare no conflict of interest.

\section{References}

1. Eaton, S.B.; Konner, M.; Shostak, M. Stone agers in the fast lane: Chronic degenerative diseases in an evolutionary perspective. Am. J. Med. 1988, 84, 739-749. [CrossRef]

2. Terry, L.L. Surgeon General's Report on Smoking and Tobacco Use. Available online: https://www.cdc.gov/ tobacco/data_statistics/sgr/index.htm (accessed on 25 June 2020).

3. Cooper, K.H. Aerobics; Bantam Books: New York, NY, USA, 1968.

4. Paffenbarger, R.S.J.; Blair, S.N.; Lee, I.M. A history of physical activity, cardiovascular health and longevity: The scientific contributions of Jeremy N Morris, DSc, DPH, FCCP. Int. J. Epidemiol. 2001, 30, 1184-1192. [CrossRef] [PubMed]

5. Paffenbarger, R.S.J.; Hale, W.E. Work activity and coronary heart mortality. N. Engl. J. Med. 1975, 292, 545-550. [CrossRef] [PubMed]

6. Paffenbarger, R.S.J.; Hyde, R.T.; Wing, A.L.; Hsieh, C.C. Physical activity, all-cause mortality and longevity of college alumni. N. Engl. J. Med. 1986, 314, 605-613. [CrossRef] [PubMed]

7. Blair, S.N.; Kungert, J.B.; Kohl, H.W.; Barlow, C.E.; Macera, C.A.; Paffenbarger Jr, R.S.; Gibbons, L.W. Influences of cardiorespiratory fitness and other precursors of cardiovascular disease and all-cause mortality in men and women. JAMA 1996, 276, 205-210. [CrossRef]

8. Lee, I.M.; Shiroma, E.J.; Lobelo, F.; Puslza, P.; Blair, S.N.; Katzmarek, P.J. Effect of physical inactivity on major non-communicable diseases worldwide: An analysis of burden of disease and life expectancy. Lancet 2012, 380, 21-27. [CrossRef]

9. O'Keefe, J.; Lavie, C.J.; Guazzi, M. Potential dangers of extreme endurance exercise: How much is too much? Prog. Cardiovasc. Dis. 2015, 57, 396-405. [CrossRef]

10. Arem, H.; Moore, S.C.; Patel, A.; Hartge, P.; Berrington de Gonzalez, A.; Visvanathan, K.; Campbell, P.T.; Freedman, M.; Weiderpass, E.; Adami, H.O.; et al. Leisure time physical activity and mortality: A detailed pooled analysis of the dose-response relationship. JAMA Intern. Med. 2015, 175, 959-967. [CrossRef]

11. Eijsvogels, T.M.; Molossi, S.; Lee, D.C.; Emery, M.S.; Thompson, P.D. Exercise at the extremes: The amount of exercise to reduce cardiovascular events. J. Am. Coll. Cardiol. 2016, 67, 316-329. [CrossRef]

12. Kraus, W.E.; Janz, K.F.; Powell, K.E.; Campbell, W.W.; Jakicic, J.M.; Troiano, R.P.; Sprow, K.; Torres, A.; Piercy, K.L. Daily step counts for measuring physical activity exposure and its relation to health. Med. Sci. Sports Exerc. 2019, 51, 1206-1212. [CrossRef]

13. Kraus, W.E.; Powell, K.E.; Haskell, W.L.; Janz, K.F.; Campbell, W.W.; Jakicic, J.M.; Troiano, R.P.; Sprow, K.; Torres, A.; Piercy, K.L. Physical activity and cardiovascular mortality and cardiovascular disease. Med. Sci. Sports Exerc. 2019, 51, 1270-1281. [CrossRef] 
14. Tudor-Locke, C.; Craig, C.L.; Aoyagi, Y.; Bell, R.C.; Croteau, K.A.; De Bourdeaudhuij, I.; Ewald, B.; Gardner, A.W.; Hatano, Y.; Lutes, L.D.; et al. How many steps/day are enough? For older adults and special populations. Int. J. Behav. Nutr. Phys. Act. 2011, 8, 1-19. [CrossRef] [PubMed]

15. Foster, C.; Porcari, J.P.; Battista, R.A.; Udermann, B.; Wright, G.; Lucia, A. The risk in exercise training. Am. J. Lifestyle. Med. 2008, 2, 279-284. [CrossRef]

16. Sanchis-Gomar, F.; Pérez, L.M.; Joyner, M.J.; Löllgen, H.; Lucia, A. Endurance exercise and the heart. Friend or foe? Sports Med. 2016, 46, 459-466. [CrossRef] [PubMed]

17. Thompson, P.D.; Franklin, B.A.; Balady, G.J.; Blair, S.N.; Corrado, D.; Estes, N.A.; Fulton, J.E.; Gordon, N.F.; Haskell, W.L.; Link, M.S.; et al. Exercise and acute cardiovascular events placing the risks into perspective: A scientific statement from the American Heart Association Council on Nutrition, Physical Activity, and Metabolism and the Council on Clinical Cardiology. Circulation 2007, 115, 2358-2368. [PubMed]

18. ACSM. ACSM's Guidelines for Exercise Testing and Prescription, 10th ed.; Wolters Kluwer: Philadelphia, PA, USA, 2017.

19. Foster, C.; Porcari, J.P.; Cress, M. Clinical exercise testing. In ACSM's Clinical Exercise Physiology; Thompson, W.R., Ed.; Wolters Kluwer: Baltimore, MD, USA, 2019; pp. 349-364.

20. Meyer, T.; Lucía, A.; Earnest, C.P.; Kindermann, W. A conceptual framework for performance diagnosis and training prescription from submaximal gas exchange parameters-theory and application. Int. J. Sports Med. 2005, 26, S38-S48. [CrossRef] [PubMed]

21. Myers, J.; Prakash, M.; Froelicher, V.; Do, D.; Partington, S.; Atwood, J.E. Exercise capacity and mortality among men referred for exercise testing. N. Engl. J. Med. 2002, 346, 793-801. [CrossRef]

22. Mark, D.B.; Shaw, L.; Harrell, F.E.; Hlatky, M.A.; Lee, K.L.; Bengtson, J.R.; McCants, C.B.; Califf, R.M.; Pryor, D.B. Prognostic value of a treadmill exercise score in outpatients with suspected coronary artery disease. N. Engl. J. Med. 1991, 325, 849-853. [CrossRef]

23. Cumming, G.R. Yield of ischaemic electrocardiograms in relation to exercise intensity in a normal population. Br. Heart J. 1972, 34, 919-923. [CrossRef]

24. Alajmi, R.A.; Foster, C.; Porcari, J.P.; Radtke, K.; Doberstein, S. Comparison of non-maximal tests for estimating exercise capacity. Kinesiology 2020, 52, 10-18. [CrossRef]

25. Mezzani, A.; Hamm, L.F.; Jones, A.M.; McBride, P.E.; Moholdt, T.; Stone, J.A.; Urhausen, A.; Williams, M.A. Aerobic exercise intensity assessment and prescription in cardiac rehabilitation: A joint position statement of the European Association for Cardiovascular Prevention and Rehabilitation, the American Association of Cardiovascular and Pulmonary Rehabilitation, and the Canadian Association of Cardiac Rehabilitation. J. Cardiopulm. Rehabil. Prev. 2012, 32, 327-350. [PubMed]

26. Seiler, $\mathrm{S}$. What is the best practiced for training intensity and duration distribution in endurance athletes? Int. J. Sports. Physiol. Perform. 2010, 5, 276-291. [CrossRef] [PubMed]

27. Myers, J.; Voodi, L.; Umann, P.A.; Froelicher, V.F. A survey of exercise testing: Methods, utilization, interpretation and safety in the VAHCS. J. Cardiopulm. Rehabil. 2000, 20, 251-258. [CrossRef]

28. Foster, C. Is there risk in exercise testing of athletes? Int. J. Sports Physiol. Perform. 2017, 12, 849-850. [CrossRef] [PubMed]

29. Eston, R.; Evans, H.; Faulkner, J.; Lambrick, D.; Al-Rahamneh, G.; Parfitt, G. A perceptually regulated, graded exercise test predicts peak oxygen uptake during treadmill exercise in active and sedentary participants. Eur. J. Appl. Physiol. 2012, 112, 3459-3468. [CrossRef] [PubMed]

30. Foster, C.; Porcari, J.P.; Doro, K.; Dubiel, J.; Engen, M.; Kolman, D.; Ault, S.; Xiong, S. Exercise prescription when there is no exercise test: The Talk Test. Kinesiology 2018, 50, 333-348.

31. Pate, R.R.; Pratt, M.; Blair, S.N.; Haskell, W.L.; Macera, C.A.; Bouchard, C.; Buchner, D.; Ettinger, W.; Heath, G.W.; King, A.C. Physical activity and public health. A recommendation from the Centers for Disease Control and Prevention and the American College of Sports Medicine. JAMA 1995, 273, 402-407. [CrossRef]

32. Haskell, W.L.; Lee, I.M.; Pate, R.R.; Powell, K.E.; Blair, S.N.; Franklin, B.A.; Macera, C.A.; Heath, G.W.; Thompson, P.D.; Bauman, A. Physical activity and public health: Updated recommendation for adults from the American College of Sports Medicine and the American Heart Association. Med. Sci. Sports Exerc. 2007, 39, 1423-1434. [CrossRef]

33. Robergs, R.A.; Landwehr, R. The surprising history of the "HRmax=220-age" equation. J. Ex. Phys. Online 2002, 5, 1-10. 
34. Katch, V.L.; Weltman, A.; Sady, S.; Freedson, P. Validity of the relative percent concept for equating training intensity. Eur. J. Appl. Physiol. Occup. Physiol. 1978, 39, 219-227. [CrossRef]

35. Sharhag-Rosenberger, F.; Meyer, T.; Gasler, N.; Faude, O.; Kindermann, W. Exercise at given percentages of $\mathrm{VO}_{2}$ max: Heterogenous metabolic responses between individuals. J. Sci. Med. Sport 2010, 13, 74-79. [CrossRef] [PubMed]

36. Sylta, Ø.; Tønnessen, E.; Hammarström, D.; Danielsen, J.; Skovereng, K.; Ravn, T.; Rønnestad, B.R.; Sandbakk, Ø.; Seiler, S. The effect of different high-intensity periodization models on endurance adaptations. Med. Sci. Sports. Exerc. 2016, 48, 2165-2174. [CrossRef] [PubMed]

37. Parfitt, G.; Evans, H.; Eston, R. Perceptually regulated training at RPE13 is pleasant and improves physical health. Med. Sci. Sports. Exerc. 2012, 44,1613-1618. [CrossRef] [PubMed]

38. Foster, C.; Lemberger, K.; Thompson, N.N.; Sennett, S.M.; Hare, J.; Pollock, M.L.; Pels, A.E.; Schmidt, D.H. Functional translation of exercise responses from graded exercise testing to exercise training. Am. Heart J. 1986, 112, 1309-1316. [CrossRef]

39. Foster, C.; Thompson, N.N.; Bales, S. Functional translation of exercise responses using combined arm-leg ergometry. Cardiology 1991, 78, 150-155. [CrossRef]

40. Foster, C.; Thompson, N.N. Functional translation of exercise test responses to recreational activities. J. Cardiopulm. Rehabil. 1991, 11, 373-377. [CrossRef]

41. Foster, C.; Porcari, J.P.; Gibson, M.; Wright, G.; Greany, J.; Talati, N.; Recalde, P. Translation of submaximal exercise test responses to exercise prescription using the Talk Test. J. Strength Cond. Res. 2009, 23, 2425-2429. [CrossRef]

42. Jeans, E.A.; Foster, C.; Porcari, J.P.; Gibson, M.; Doberstein, S. Translation of exercise testing to exercise prescription using the Talk Test. J. Strength Cond. Res. 2011, 25, 590-596. [CrossRef]

43. Woltmann, M.L.; Foster, C.; Porcari, J.P.; Camic, C.L.; Dodge, C.; Haible, S.; Mikat, R.P. Evidence that the Talk Test can be used to regulated exercise intensity. J. Strength Cond. Res. 2015, 29, 1248-1254. [CrossRef]

44. Lyon, E.; Menke, M.; Foster, C.; Porcari, J.P.; Gibson, M.; Bubbers, T. Translation of incremental Talk Test responses to steady-state exercise training intensity. J. Cardiopulm. Rehabil. 2014, 34, 271-275. [CrossRef]

45. De Koning, J.J.; Noorhof, D.A.; Uitslag, T.P.; Gilart, R.E.; Dodge, C.; Foster, C. An approach to estimating gross efficiency during high intensity exercise. Int. J. Sports Physiol. Perform. 2013, 8, 682-684. [CrossRef] [PubMed] 